\title{
Relationship of common expression quantitative trait loci genes to the immune system
}

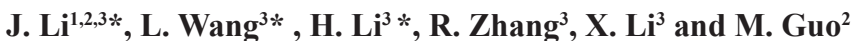

${ }^{1}$ School of Life Science and Technology, Harbin Institute of Technology, Harbin, China

${ }^{2}$ School of Computer Science and Technology, Harbin Institute of Technology, Harbin, China

${ }^{3}$ Department of Bioinformatics and Computer, Harbin Medical University, Harbin, China

*These authors contributed equally to this study

Corresponding authors: X. Li / M. Guo

E-mail: lixia6@yahoo.com / maozuguo@hit.edu.cn

Genet. Mol. Res. 12 (4): 6546-6553 (2013)

Received January 15, 2013

Accepted September 20, 2013

Published December 11, 2013

DOI http://dx.doi.org/10.4238/2013.December.11.6

\begin{abstract}
Tissue-specific expression quantitative trait loci (eQTLs) are always linked to specific diseases. In this study, we focused on eQTLs common to multiple tissues and explored their functional mechanisms in disease for the first time. We found 11 common eQTL genes among multiple tissues. Five genes were validated through a genome-wide association study, 3 genes were validated using the Online Mendelian Inheritance in Man database, and the others were validated through text mining. Most of these disorders were related to the systemic immune system. In functional analyses using Gene Ontology and the Kyoto Encyclopedia of Genes and Genomes, these common eQTL genes were enriched in biological processes and pathways mostly related to the
\end{abstract}


immunity. Therefore, we believe that these common eQTL genes are related to immune system.

Key words: Multiple tissues; Expression quantitative trait locus; Immune system

\section{INTRODUCTION}

Recent developments in high-throughput genotyping technology, next-generation sequencing technologies, and genome-wide association studies (GWAS) have been widely applied to find loci and genes related to complex diseases such as coronary heart disease (McPherson et al., 2007), type 2 diabetes (Sladek et al., 2007), Crohn's disease (WTCCC, 2007), and host control of human immunodeficiency virus (HIV) type 1 (Fellay et al., 2007). However, explorations of the relationship between DNA sequence variations and phenotypes in GWAS ignore many middle molecular phenotypes, such as gene transcript levels and protein expression levels. These molecular phenotypes influenced by DNA sequence variation may also induce disease (Schadt et al., 2003; Nicolae et al., 2010). Expression quantitative trait locus (eQTL) mapping is a technique that uses genomic variation to explain expression traits. Using the level of gene expression as a middle molecular phenotype between genetic variation and clinical phenotypes in eQTLs, it provides a new way to mine risk genes and their functional mechanisms in studies of complex traits, especially complex diseases (Schadt et al., 2003; Nicolae et al., 2010). Therefore, integration of eQTL and GWAS genetic approaches not only reduce the dimensions of risk single-nucleotide polymorphism (SNP) loci in GWAS, but also effectively locate complex disease susceptibility genes and assist in functional verification of candidate genes.

Nica et al. (2011) and van Nas et al. (2010) have proven that tissue-specific eQTLs are always linked to specific diseases, so most eQTL studies use specific tissue samples - such as liver tissue samples for cirrhosis (Schadt et al., 2008) and brain tissue samples for Alzheimer's disease (Webster et al., 2009) - to investigate a disease. In this study, we focused on the common eQTLs among multiple tissues and explored the functional mechanisms of these common eQTLs in disease processes for the first time. We integrated 5 eQTL analyses from various tissues, found 11 significant common eQTL genes, and performed functional analysis, such as validation through GWAS, Online Mendelian Inheritance in Man (OMIM), text mining, Gene Ontology (GO), and Kyoto Encyclopedia of Genes and Genomes (KEGG) functional enrichment analysis.

\section{MATERIAL AND METHODS}

We used 5 eQTLs datasets from liver tissue samples (Schadt et al., 2008), normal brain tissue samples (Webster et al., 2009), cohort studies of Alzheimer's disease brain tissue samples (Myers et al., 2007), lymphoblastoid cell line samples (Montgomery et al., 2010), and monocyte samples (Zeller et al., 2010). Genome-wide genotyping and gene expression data are present in the sets. Following the statistical methods, significant thresholds, and multiple testing correction methods in the original literature, we selected significant eQTLs in these datasets for subsequent analyses. Detailed information for these datasets is shown in Table 1. 
J. Li et al.

6548

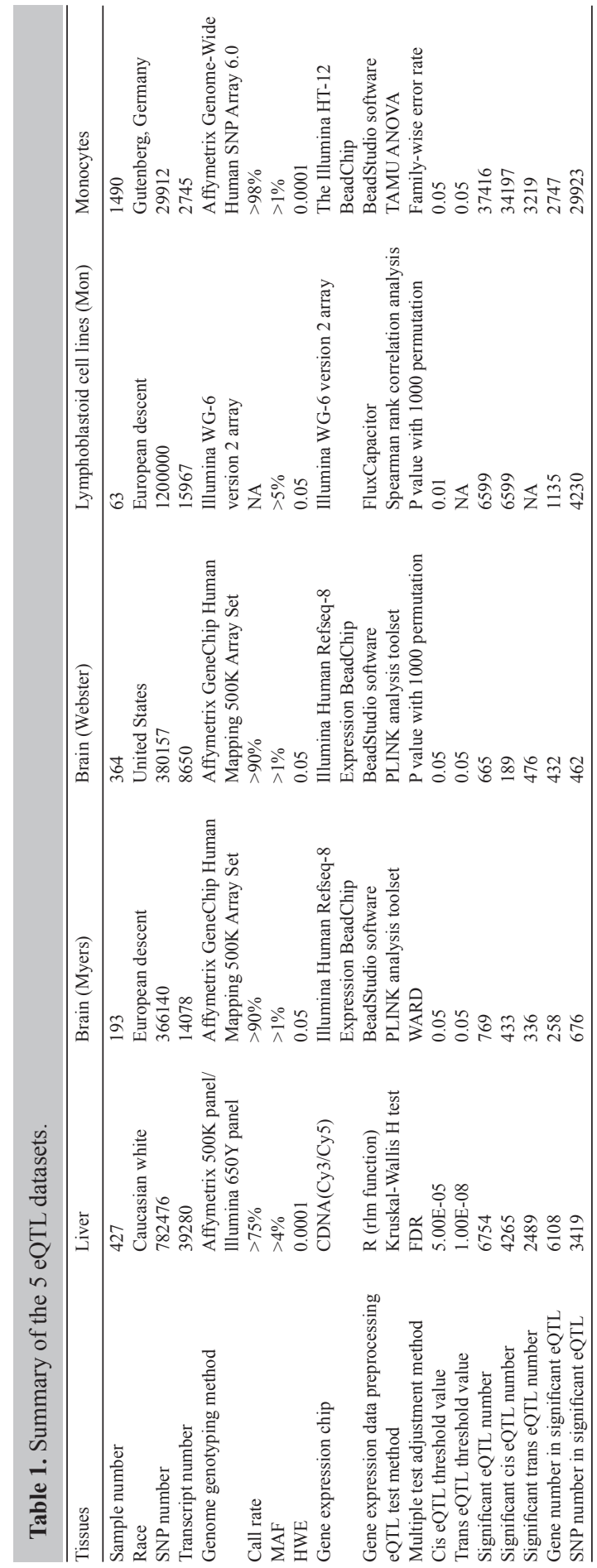


We unified a gene to be the official gene symbol. We directly extracted the data from the liver tissue dataset and 2 brain tissue datasets, as they used official gene symbols. In the data from the lymphoblastoid cell line samples, we used the David Gene ID Conversion Tool (Huang et al., 2009) to convert the Ensemble gene IDs to their corresponding official gene symbols; in the data of the monocyte samples, we extracted the official gene symbol data from the Sqlite3Explorer database provided in the original literature. We used reference SNP identifiers to determine SNPs.

We divided the significant eQTLs into cis and trans eQTLs. Molecular variation located on or near the genomic position of the regulated gene (usually within the selected gene or the range of less than $1 \mathrm{Mb}$ from the $5^{\prime}$ or $3^{\prime}$ end) was identified as a cis eQTL; otherwise it was labeled a trans eQTL (Rockman and Kruglyak, 2006). In this study, we set $1 \mathrm{Mb}$ as the threshold to separate cis and trans eQTLs. We only selected the cis eQTLs for subsequent analyses, as they have higher credibility and a more direct mechanism of action than that of trans eQTLs.

\section{RESULTS}

\section{Intersection of the 5 eQTL gene sets}

Because the 5 datasets used different chips and the diversities of the measured SNPs are large, we could not directly screen common SNPs among them. However, the most frequently detected genes are common, so we focused on the intersection of genes regulated by SNPs (said eQTL genes) among various tissues. Two datasets of brain tissue samples were included, so we first combined them and identified $658 \mathrm{eQTL}$ genes. We then intersected them with 3 additional eQTL gene datasets to obtain a final 11 common eQTL genes among 4 tissues. These genes are listed in Table 2.

Table 2. Number of eSNPs in the 5 datasets and SNPs located on the genes.

\begin{tabular}{lccc}
\hline Gene symbol & Number of eSNPs in the 5 datasets & Number of SNPs located on the gene & Number of joint SNPs \\
\hline ATPIF1 & 10 & 71 & 1 \\
CWF19L1 & 27 & 271 & 2 \\
H1F0 & 15 & 63 & 0 \\
LRRC14 & 11 & 83 & 2 \\
HLA-A & 177 & 2750 & 1 \\
HLA-B & 311 & 2306 & 4 \\
HLA-DRB1 & 453 & 4933 & 1 \\
RPS26 & 35 & 77 & 1 \\
SIRT1 & 13 & 318 & 0 \\
SLC4A7 & 14 & 954 & 0 \\
ZNF266 & 47 & 406 & 8 \\
\hline
\end{tabular}

\section{Validation through GWAS, OMIM, and text mining}

eQTL genes have been proven to be strongly related to genes found through GWAS, so we can validate more accurate disease-related genes by combining eQTL and GWAS (Nicolae et al., 2010). We took these 11 genes into the GWAS catalog (Hindorff et al., 2009, 2011) to search for SNPs using a default threshold $\mathrm{P}$ value of $10^{-5}$. The results are shown in Table 3. We confirmed that 5 of these 11 genes are related to complex diseases through GWAS. In particular, the strongest SNP loci rs2523608 and rs2523590 on major histocompat- 
ibility complex, class I, B (HLA-B) were associated with HIV-1 control (Pelak et al., 2010; Pereyra et al., 2010), and they are also linked to HLA-B gene expression in lymphoid cell lines (Montgomery et al., 2010). Some research has found that a variety of HLA-B gene subtypes is related to the progression of HIV infection (Gao et al., 2001; Carrington and O'Brien, 2003). We also confirmed that loci rs9268853 and rs9271100, which are associated with ulcerative colitis (Pereyra et al., 2010) and systemic lupus erythematosus (Han et al., 2009; Alcina et al., 2012), affect the expression of HLA class II, DR beta 1 (HLA-DRB1) in lymphoid cell lines (Montgomery et al., 2010). The results showed that the eQTL genes shared among tissues are consistent with disease risk genes and loci in GWAS.

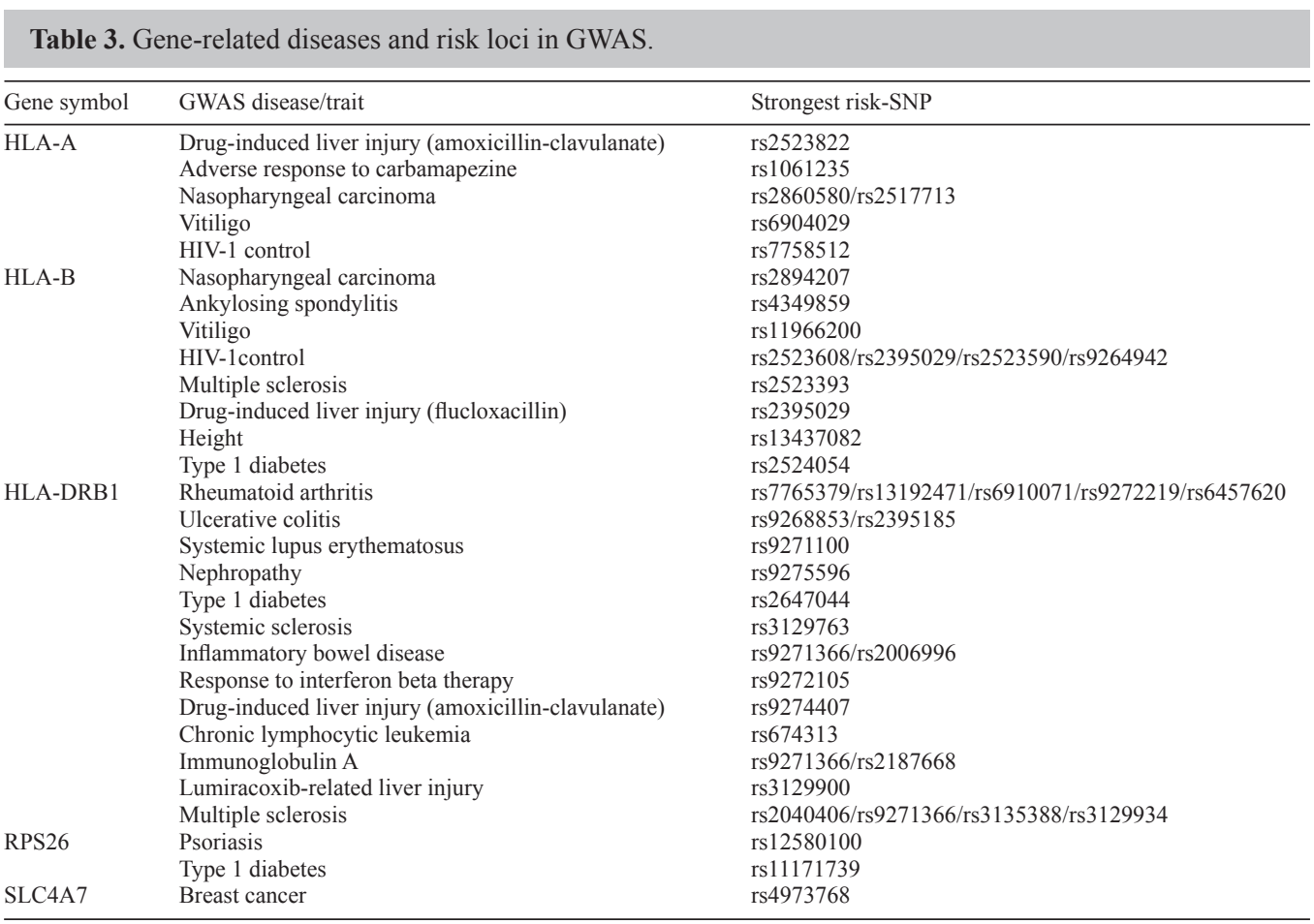

To explore further whether the mutations or deletions of these genes lead to genetic diseases, we queried these genes in OMIM (Hamosh et al., 2000). The results are shown in Table 4. We used ankylosing spondylitis as an example. Several studies (Monnet et al., 2004; $\mathrm{Gu}$ et al., 2009) have shown that HLA-B27 is a risk factor for ankylosing spondylitis. Because the HLA system is genetically determined, the genetic pathogenesis of ankylosing spondylitis is an important factor. In addition to genetic factors, immune disorders and systemic inflammation of the body are related to spondyloarthropathy. Furthermore, some research has confirmed that HLA-B27 is associated with other immune system and inflammatory diseases, such as psoriasis (Sheehan, 2004), acute anterior uveitis (Chang et al., 2005), and Crohn's disease (Purrmann et al., 1988; Khan, 1989).

We adopted text mining for another 7 genes and confirmed that ATPase inhibitory factor 1 is related to tumor formation (Sanchez-Cenizo et al., 2010); H1 histone family member 0 
is related to systemic lupus erythematosus (Armananzas et al., 2009); Sirtuin 1 forms a pathway with microRNA-134 to regulate memory and plasticity (Gao et al., 2010) and is related to insulin resistance (Liang et al., 2009) and cancer, as demonstrated previously in a mouse model (Herranz et al., 2010); and a segment of leucine rich repeat containing 14 is related to bladder cancer (Rothman et al., 2010), schizophrenia, bipolar disorder (Wang et al., 2010), and attention deficit hyperactivity disorder (Lasky-Su et al., 2008). The results of analyses showed that the $11 \mathrm{eQTL}$ genes common to multiple tissues were related with a variety of diseases or biological traits - mostly disorders of the systemic immune system such as cancers and autoimmune diseases.

\section{Table 4. Results queried in OMIM of the eQTL genes.}

\begin{tabular}{llcc}
\hline Gene/locus & Phenotype & Gene/locus MIM number & Location \\
\hline HLA-A & Severe cutaneous adverse reaction & 142800 & $6 \mathrm{p} 21.3$ \\
HLA-B & Abacavir hypersensitivity & 142830 & $6 \mathrm{p} 21.33$ \\
& Drug-induced liver injury due to flucloxacillin & & \\
& Spondyloarthropathy & & \\
& Stevens-Johnson syndrome, carbamazepine-induced & & \\
RPS26 & Synovitis, chronic & 603701 & $12 \mathrm{q} 13.2$ \\
\hline
\end{tabular}

\section{GO and KEGG functional enrichment analysis}

To explore further the biological processes in which these 11 genes are involved, we performed GO and KEGG functional enrichment analysis using the DAVID Functional Annotation Tool (Huang et al., 2009). The significant results are detailed in Table 5 with a P value of $<0.05$ as the threshold.

\begin{tabular}{|c|c|c|c|}
\hline GO ID or KEGG ID & Term name & Genes & $P$ value \\
\hline GO:0019882 & Antigen processing and presentation & HLA-A, HLA-B, HLA-DRB1 & 0.001 \\
\hline GO:0002474 & Antigen processing and presentation of peptide antigen via MHC class I & HLA-A, HLA-B & 0.010 \\
\hline GO:0048002 & Antigen processing and presentation of peptide antigen & HLA-A, HLA-B & 0.016 \\
\hline GO:0051346 & Negative regulation of hydrolase activity & ATPIF1, SIRT1 & 0.028 \\
\hline GO:0045934 & Negative regulation of nucleobase, nucleoside, nucleotide, and nucleic acid & ATPIF1, SIRT1, RPS26 & 0.034 \\
\hline GO:0051172 & Negative regulation of nitrogen compound metabolic process & ATPIF1, SIRT1, RPS26 & 0.035 \\
\hline hsa05330 & Allograft rejection & HLA-A, HLA-B, HLA-DRB1 & $1.46 \mathrm{E}-4$ \\
\hline hsa05332 & Graft-versus-host disease & HLA-A, HLA-B, HLA-DRB1 & $1.71 \mathrm{E}-4$ \\
\hline hsa04940 & Type I diabetes mellitus & HLA-A, HLA-B, HLA-DRB1 & $1.99 \mathrm{E}-4$ \\
\hline hsa05320 & Autoimmune thyroid disease & HLA-A, HLA-B, HLA-DRB1 & $2.94 \mathrm{E}-4$ \\
\hline hsa05416 & Viral myocarditis & HLA-A, HLA-B, HLA-DRB1 & $5.72 \mathrm{E}-4$ \\
\hline hsa04612 & Antigen processing and presentation & HLA-A, HLA-B, HLA-DRB1 & 7.81E-4 \\
\hline hsa04514 & Cell adhesion molecules & HLA-A, HLA-B, HLA-DRB1 & $1.09 \mathrm{E}-3$ \\
\hline
\end{tabular}

In the $\mathrm{GO}$ analysis, these genes were enriched in the categories antigen processing and presentation; negative regulation of hydrolase activity; negative regulation of nucleobase, nucleoside, nucleotide, and nucleic acid; and negative regulation of nitrogen compound metabolic process. In the KEGG pathway analysis, these genes, especially HLA-A, HLA-B, and HLA-DRB1 were enriched in the categories allograft rejection, graft-versus-host disease, type I diabetes mellitus, autoimmune thyroid disease, viral myocarditis, and antigen processing and presentation. Clearly, 
these biological processes and pathways are primarily related to the immune system.

\section{DISCUSSION}

In this study, we analyzed 11 common eQTL genes among 5 tissues. Among them, 5 genes were validated to be associated with complex diseases through GWAS, 3 genes were validated to be associated with diseases or biological traits through OMIM analysis, and others were validated with text mining. Most of the associations were disorder related to the systemic immune system - for example, cancers and autoimmune diseases. In the GO and KEGG functional enrichment analyses, these genes - especially HLA-A, HLA-B, and HLA-DRB1 - were enriched in biological processes and pathways such as antigen processing and presentation, allograft rejection, and autoimmune thyroid disease that are mostly related to the immune system.

Therefore, we believe that these eQTL genes common to multiple tissues are most likely to be related to immune system. However, few genome-wide eQTL analysis datasets are available, and we used only 1 or 2 datasets for each tissue. When more genome-wide eQTL datasets are available, we will perform deeper analysis for common eQTL genes.

\section{Conflicts of interest}

The authors declare no conflicts of interest.

\section{ACKNOWLEDGMENTS}

Research partially supported by the National Natural Science Foundation of China (grants \#61300116, \#61271346, \#91335112, \#60932008, and \#61172098), the Specialized Research Fund for the Doctoral Program of Higher Education of China (grant \#20112302110040), the Natural Science Foundation of Heilongjiang Province (grant \#QC2013C063), the Fund of Heilongjiang Education Department (grants \#12531298 and \#12531299), the Fund of Heilongjiang Health Department (grants \#2011-204 and \#2011-251).

\section{REFERENCES}

Alcina A, Abad-Grau Mdel M, Fedetz M, Izquierdo G, et al. (2012). Multiple sclerosis risk variant HLA-DRB1*1501 associates with high expression of DRB1 gene in different human populations. PLoS One 7: e29819.

Armananzas R, Calvo B, Inza I, Lopez-Hoyos M, et al. (2009). Microarray analysis of autoimmune diseases by machine learning procedures. IEEE Trans. Inf. Technol. Biomed. 13: 341-350.

Carrington M and O'Brien SJ (2003). The influence of HLA genotype on AIDS. Annu. Rev. Med. 54: 535-551.

Chang JH, McCluskey PJ and Wakefield D (2005). Acute anterior uveitis and HLA-B27. Surv. Ophthalmol. 50: 364-388.

Fellay J, Shianna KV, Ge D, Colombo S, et al. (2007). A whole-genome association study of major determinants for host control of HIV-1. Science 317: 944-947.

Gao J, Wang WY, Mao YW, Graff J, et al. (2010). A novel pathway regulates memory and plasticity via SIRT1 and miR134. Nature 466: 1105-1109.

Gao X, Nelson GW, Karacki P, Martin MP, et al. (2001). Effect of a single amino acid change in MHC class I molecules on the rate of progression to AIDS. N. Engl. J. Med. 344: 1668-1675.

Gu J, Huang J, Li C, Zhao L, et al. (2009). Association of chromosome 2q36.1-36.3 and autosomal dominant transmission in ankylosing spondylitis: results of genetic studies across generations of Han Chinese families. J. Med. Genet. 46: 657-662.

Hamosh A, Scott AF, Amberger J, Valle D, et al. (2000). Online mendelian inheritance in man (OMIM). Hum. Mutat. 15: 57-61. 
Han JW, Zheng HF, Cui Y, Sun LD, et al. (2009). Genome-wide association study in a Chinese Han population identifies nine new susceptibility loci for systemic lupus erythematosus. Nat. Genet. 41: 1234-1237.

Herranz D, Munoz-Martin M, Canamero M, Mulero F, et al. (2010). Sirt1 improves healthy ageing and protects from metabolic syndrome-associated cancer. Nat. Commun. 1: 3 .

Hindorff L, MacArthur J, Wise A and Junkins H (2011). A Catalog of Published Genome-Wide Association Studies. Available at [www.genome.gov/gwastudies]. Accessed June 19, 2012.

Hindorff LA, Sethupathy P, Junkins HA, Ramos EM, et al. (2009). Potential etiologic and functional implications of genome-wide association loci for human diseases and traits. Proc. Natl. Acad. Sci. U. S. A. 106: 9362-9367.

Huang dW, Sherman BT and Lempicki RA (2009). Systematic and integrative analysis of large gene lists using DAVID bioinformatics resources. Nat. Protoc. 4: 44-57.

Khan MA (1989). HLA-B27 and B12(B44) in Crohn's disease with ankylosing spondylitis. J. Rheumatol. 16: 851-852.

Lasky-Su J, Neale BM, Franke B, Anney RJ, et al. (2008). Genome-wide association scan of quantitative traits for attention deficit hyperactivity disorder identifies novel associations and confirms candidate gene associations. Am. $J$. Med. Genet. B: Neuropsychiatr. Genet. 147B: 1345-1354.

Liang F, Kume S and Koya D (2009). SIRT1 and insulin resistance. Nat. Rev. Endocrinol. 5: 367-373.

McPherson R, Pertsemlidis A, Kavaslar N, Stewart A, et al. (2007). A common allele on chromosome 9 associated with coronary heart disease. Science 316: 1488-1491.

Monnet D, Breban M, Hudry C, Dougados M, et al. (2004). Ophthalmic findings and frequency of extraocular manifestations in patients with HLA-B27 uveitis: a study of 175 cases. Ophthalmology 111: 802-809.

Montgomery SB, Sammeth M, Gutierrez-Arcelus M, Lach RP, et al. (2010). Transcriptome genetics using second generation sequencing in a Caucasian population. Nature 464: 773-777.

Myers AJ, Gibbs JR, Webster JA, Rohrer K, et al. (2007). A survey of genetic human cortical gene expression. Nat. Genet. 39: 1494-1499.

Nica AC, Parts L, Glass D, Nisbet J, et al. (2011). The architecture of gene regulatory variation across multiple human tissues: the MuTHER study. PLoS Genet. 7: e1002003.

Nicolae DL, Gamazon E, Zhang W, Duan S, et al. (2010). Trait-associated SNPs are more likely to be eQTLs: annotation to enhance discovery from GWAS. PLoS. Genet. 6: e1000888.

Pelak K, Goldstein DB, Walley NM, Fellay J, et al. (2010). Host determinants of HIV-1 control in African Americans. $J$. Infect. Dis. 201: 1141-1149.

Pereyra F, Jia X, McLaren PJ, Telenti A, et al. (2010). The major genetic determinants of HIV-1 control affect HLA class I peptide presentation. Science 330: 1551-1557.

Purrmann J, Zeidler H, Bertrams J, Juli E, et al. (1988). HLA antigens in ankylosing spondylitis associated with Crohn's disease. Increased frequency of the HLA phenotype B27, B44. J. Rheumatol. 15: 1658-1661.

Rockman MV and Kruglyak L (2006). Genetics of global gene expression. Nat. Rev. Genet. 7: 862-872.

Rothman N, Garcia-Closas M, Chatterjee N, Malats N, et al. (2010). A multi-stage genome-wide association study of bladder cancer identifies multiple susceptibility loci. Nat. Genet. 42: 978-984.

Sanchez-Cenizo L, Formentini L, Aldea M, Ortega AD, et al. (2010). Up-regulation of the ATPase inhibitory factor 1 (IF1) of the mitochondrial H+-ATP synthase in human tumors mediates the metabolic shift of cancer cells to a Warburg phenotype. J. Biol. Chem. 285: 25308-25313.

Schadt EE, Monks SA, Drake TA, Lusis AJ, et al. (2003). Genetics of gene expression surveyed in maize, mouse and man. Nature 422: 297-302.

Schadt EE, Molony C, Chudin E, Hao K, et al. (2008). Mapping the genetic architecture of gene expression in human liver. PLoS. Biol. 6: e107.

Sheehan NJ (2004). The ramifications of HLA-B27. J. R. Soc. Med. 97: 10-14.

Sladek R, Rocheleau G, Rung J, Dina C, et al. (2007). A genome-wide association study identifies novel risk loci for type 2 diabetes. Nature 445: 881-885.

van Nas A, Ingram-Drake L, Sinsheimer JS, Wang SS, et al. (2010). Expression quantitative trait loci: replication, tissueand sex-specificity in mice. Genetics 185: 1059-1068.

Wang KS, Liu XF and Aragam N (2010). A genome-wide meta-analysis identifies novel loci associated with schizophrenia and bipolar disorder. Schizophr. Res. 124: 192-199.

Webster JA, Gibbs JR, Clarke J, Ray M, et al. (2009). Genetic control of human brain transcript expression in Alzheimer disease. Am. J. Hum. Genet. 84: 445-458.

WTCCC (2007). Genome-wide association study of 14,000 cases of seven common diseases and 3,000 shared controls. Nature 447: 661-678.

Zeller T, Wild P, Szymczak S, Rotival M, et al. (2010). Genetics and beyond--the transcriptome of human monocytes and disease susceptibility. PLoS One 5: e10693. 